\title{
Apoptosis regulation at the mitochondria membrane level
}

Shashank Dadsena ${ }^{\dagger, 1}$, Louise E. King ${ }^{\dagger, 1}$, Ana J. García-Sáez ${ }^{{ }^{*}}$

${ }^{1}$ Institute for Genetics, CECAD Research Center, University of Cologne, Germany.

${ }^{\dagger}$ equal contribution

*correspondence to: ana.garcia@uni-koeln.de

\section{ORCID:}

Shashank Dadsena 0000-0002-0227-5900

Louise E. King 0000-0003-2453-8887

Ana J. García-Sáez 0000-0002-3894-5945

\section{Highlights}

- MOM permeabilization is a key event in the cell suicidal program

- Bax and Bak are the master effectors of MOMP and are regulated by Bcl-2 interplay

- Bcl-2 family protein interactions are partly regulated by association with the MOM

- MOM lipids and proteins like VDAC, HK and Mtch2 regulate Bax/Bak activation

- Including MOM lipid/protein interactions in $\mathrm{BH} 3$-mimetic design may improve efficacy

\begin{abstract}
Mitochondrial outer membrane permeabilization (MOMP) is a key checkpoint in apoptosis that activates the caspase cascade and irreversibly causes the majority of cells to die. The proteins of the Bcl-2 family are master regulators of apoptosis that form a complex interaction network within the mitochondrial membrane that determines the induction of MOMP. This culminates in the activation of the effector members Bax and Bak, which permeabilize the mitochondrial outer membrane to mediate MOMP. Although the key role of Bax and Bak has been established, many questions remain unresolved regarding molecular mechanisms that control the
\end{abstract}


apoptotic pore. In this review, we discuss the recent progress in our understanding of the regulation of Bax/Bak activity within the mitochondrial membrane.

Keywords: Apoptosis, BCL-2 proteins, MOMP, membrane dynamics, protein-protein interaction, lipids

\section{Abbreviations}

Bad - Bcl-2-associated death promoter

Bak - Bcl-2 homologous antagonist/killer

Bax - Bcl-2-associated $X$ protein

Bcl-2 - B-cell lymphoma 2

Bcl-W - Bcl-2-like protein 2

$\mathrm{Bcl}-\mathrm{XL}$ - B-cell lymphoma-extra large

$\mathrm{BH}$ - Bcl-2 homology

Bid - $\mathrm{BH} 3$ interacting-domain death agonist

Bik - Bcl-2-interacting killer

Bim - Bcl-2-like protein 11

$\mathrm{Bmf}$ - Bcl-2-modifying factor

Bok - Bcl-2 related ovarian killer

$\mathrm{CL}$ - Cardiolipin

DRP1 - Dynamin-related protein 1

EL - Endosomes/lysosomes

FCCS - Fluorescence cross-correlation spectroscopy

FRET - Förster resonance energy transfer

GUV - Giant unilamellar vesicle

HK - Hexokinase

Hrk - Harakiri

IP3R - Inositol 1,4,5-trisphosophate receptor

MAM - Mitochondrial associated membrane

MAPL - Mitochondrial-anchored protein/SUMO ligase

Mcl-1 - Induced myeloid leukemia cell differentiation protein

MERC - Mitochondria-ER contact sites

MFN - Mitofusin 
MOM - Mitochondrial outer membrane

MOMP - Mitochondrial outer membrane permeabilisation

Mtch2 - Mitochondrial carrier homolog 2

Noxa - Phorbol-12-myristate-13-acetate-induced protein 1

OPA1 - Optic atrophy 1

OXPHOS - Oxidative phosphorylation

PINK1 - PTEN-induced Kinase 1

Puma - p53 upregulated modulator of apoptosis

SMAC - Second mitochondria-derived activator of caspases

STED - Stimulated emission depletion

VDAC2 - Voltage-dependent anion channel 2 


\section{Introduction to apoptosis regulation by the Bcl-2 proteins}

Maintaining tight control over cell homeostasis via apoptosis is critical in the prevention of diseases such as cancer or neurodegenerative disorders [1]. The Bcl-2 family proteins are the master regulators of this process. Their interactions with each other in response to a pro-apoptotic stimulus dictate whether mitochondrial outer membrane (MOM) permeabilization takes place, releasing factors from the intermembrane space such as cytochrome $c$ to form the apoptosome, which leads to the activation caspases and to the killing of the cell [2]. In general, MOM permeabilization (MOMP) is considered the "point of no return" in apoptosis induction, with cells committed to die after this threshold is reached.

The Bcl-2 family comprises approximately 20 proteins having either pro- or antiapoptotic roles [3]. All members of the family share at least one of four regions of homology within their sequence, termed $\mathrm{Bcl}-2$ homology $(\mathrm{BH})$ domains 1 to 4 . Proteins within the family are classified into groups depending on their structure and function: the anti-apoptotic proteins (including Bcl-2, Bcl-XL, Bcl-W, Mcl-1 and A1) present all four $\mathrm{BH}$ domains, the multi-domain pro-apoptotic effectors (Bax, Bak and Bok) also contain $\mathrm{BH} 1$ to 4 , and the $\mathrm{BH} 3$-only pro-apoptotic proteins (including Bad, Bid, Bim, Puma, Noxa, Bik, Hrk and Bmf) contain instead only the BH3 domain [4]. Of the four $\mathrm{BH}$ domains, the $\mathrm{BH} 3$ domain is crucial in regulating interactions between proteins within the family, allowing then to carry out their pro- or anti-apoptotic roles by regulating the formation of Bax/Bak-dependent pores in the MOM. The $\mathrm{BH} 3$ domain interacts with a hydrophobic groove on the surface of Bax and Bak, composed of residues within the $\alpha 2$ to $\alpha 5$ helix and residues in the $\alpha 8$ helix [5]. Similar hydrophobic grooves are also present in the anti-apoptotic proteins where they engage the BH3-only proteins. The mechanism by which Bax and Bak are activated to form pores in the MOM has been an area of debate for a number of years. In healthy cells, Bax and Bak are inactive, with Bak localised at the MOM, and Bax mainly cytosolic due to its constitutive retrotranslocation from the mitochondria via anti-apoptotic Bcl-XL [6]-[8]. Sequestration of Bax and Bak by anti-apoptotic Bcl-2 proteins is therefore a crucial mechanism for maintaining the integrity of the MOM. How Bax and Bak become activated in the presence of a pro-apoptotic stimulus is not fully clear, with multiple models of activation proposed. 
The "direct activation" model posits that Bax and Bak are activated via direct interaction with a subclass of BH3-only proteins, the "direct activators" (tBid, Bim, Puma), which bind with their $\mathrm{BH} 3$ domain into the hydrophobic groove of Bax and Bak to induce a conformational change and subsequent activation [9]. The remaining BH3-only proteins act as "sensitisers", binding to anti-apoptotic to proteins to prevent them inhibiting both the direct activators and Bax/Bak (Fig. 1a). [10], [11]. Difficulties in detecting the interactions between the direct-activators and Bax/Bak created the hypothesis that this activation occurred in a "hit and run" manner, with transient interactions sufficient [12]. A second model later challenged the direct activation model when it was discovered that in the absence of tBid, Bim and Puma, sensitiser BH3-only proteins can activate Bax/Bak [13]. The "indirect activation" or "displacement" model is formed on the basis that Bax/Bak are constitutively active, but are kept under control via inhibition by anti-apoptotic proteins (Fig. 1b). The BH3only proteins all thus serve the same function of displacing Bax/Bak from the antiapoptotics to permeabilise the MOM. Live cell studies demonstrating Bax is in dynamic equilibrium between mitochondria and cytosol, constantly removed from the MOM by Bcl-XL strengthened this argument [6]-[8].

A significant factor both models fail to address is the role of the mitochondrial membrane. Multiple studies have shown that various mitochondrial lipids and proteins interact with $\mathrm{Bcl}-2$ family proteins in both healthy and apoptotic cells [14][18]. This led to the creation of a third model of apoptosis activation, the "embedded together" model (Fig. 1c). This model identifies the insertion of Bax and Bak into the MOM as a prerequisite for membrane permeabilization, and acknowledges the role of the membrane in causing conformational changes in Bcl-2 proteins that ultimately regulate their function [19]. Using a minimal in vitro system the stepwise activation of Bax via tBid was elucidated, with the insertion of both tBid and Bax into the membrane being essential in membrane permeabilization [20]. Bcl-XL is also capable of inserting into the MOM, but unlike Bax, cannot form long-lived pores to induce MOMP, but can inhibit Bax oligomerization [21], [22]

Therefore, as research progresses, it is important to consider a more integrated approach in which factors from all models are relevant in the induction of apoptosis. Whilst Bax and Bak have the ability to autoactivate, they can also be activated by BH3-only proteins to initiate MOMP, and the conformational changes required to induce pore formation are dependent on interaction with the MOM (Fig. 2a). As 
discussed below, recent findings using multiple systems and advanced techniques are strengthening the case for this integrated model of apoptosis regulation.

\section{Regulation of Bax and Bak by the Bcl-2 network}

\section{The "sensitive" subject of the Bax activation in the membrane}

The classical view of "activator" and "sensitiser" BH3-only proteins having distinct roles in the initiation of apoptosis is based on their specific interactions with either anti-apoptotic or multi-domain $\mathrm{Bcl}-2$ proteins, with differing binding affinities based on specificity between BH3 domains [3], [23]. Sensitisers, as their name suggest, increase the susceptibility of cells to a pro-apoptotic stimulus by binding to, and sequestering, anti-apoptotic Bcl-2 proteins to free up the direct-activators. This set of interactions is a complex balancing act, as both the binding affinities and comparative levels of pro- and anti-apoptotic proteins in the cell dictate whether the balance tips in favor of apoptosis [24]. In more recent years, the direct and indirect models of Bax and Bak activation have been shown to be oversimplified, with the categorization of activators and sensitisers becoming less distinct as their roles in regulating Bax and Bak overlap [25], [26]. An emerging factor in BH3-only protein interactions with antiapoptotic Bcl-2 proteins is exactly how protein binding between cytosol and mitochondria differ, and what role mitochondrial lipids and proteins play in regulating $\mathrm{Bcl}-2$ protein interactions to initiate MOMP.

BH3-only proteins inhibit anti-apoptotic proteins by binding the canonical hydrophobic groove of the anti-apoptotics via four conserved hydrophobic residues in their $\mathrm{BH} 3$ domain [27]. More recent studies have demonstrated that this binding is more complex than a single interacting region, as the interaction is strengthened by the $\mathrm{BH} 3$ domain binding a further interaction site. This is exemplified when mutation of two key residues in the $\mathrm{BH} 3$ domain of $\mathrm{BH} 3$-only proteins to alanine (termed $2 \mathrm{~A}$ mutants) completely abolish FRET-measured interactions of Bid and Bad with antiapoptotic proteins, but not Bim [28]. Whilst the overall level of interaction is reduced, $\mathrm{Bim}$ is able to maintain some level of interaction with $\mathrm{Bcl}-\mathrm{XL}$ when these residues are mutated. This can be explained by a recent study by Liu and colleagues in which Bim has been shown to not only bind $\mathrm{Bcl}-\mathrm{XL}$ and $\mathrm{Bcl}-2$ via its $\mathrm{BH} 3$ domain, but also to be further stabilized via residues in its $C$-terminal sequence, originally thought to function solely as a membrane tethering sequence [29]. Deletion of this $C$-terminal sequence 
not only prevents Bim localizing to the MOM, but also reduces the binding affinity between BimL and $\mathrm{Bcl}-\mathrm{XL}$ in the presence of mitochondria in vitro. This observation suggests that the $C$-terminus of Bim does not completely insert into the lipid bilayer of membranes, but rather residues that interact with $\mathrm{Bcl}-\mathrm{XL}$ are on the cytoplasmic face of the membrane. Combined with the canonical BH3 domain, this "double-bolt lock" interaction increases the stability of the Bim-Bcl-XL interaction at the MOM. Furthermore, the use of Bid chimeras where the Bid $\mathrm{BH} 3$ domain is replaced with the $\mathrm{BH} 3$ domain of other $\mathrm{BH} 3$-only proteins has also highlighted the importance of membrane localization in the interaction between $\mathrm{BH} 3-$ only and anti-apoptotic proteins [30]. When anti-apoptotic proteins are C-terminally truncated and therefore unable to localize to mitochondria, their $\mathrm{BH} 3$ binding specificity is altered. In the absence of mitochondria, the truncated anti-apoptotic proteins failed to immunoprecipitate significantly with the chimeras. Therefore, whilst the $\mathrm{BH} 3$ domain is crucial in the interaction, the interaction between the $\mathrm{C}$-terminus of the proteins and the MOM is also important.

One key impact of this finding is how this alternative interaction site affects the ability of other $\mathrm{BH} 3-$ only proteins, or indeed drugs mimicking these proteins, to displace Bim from $\mathrm{Bcl}-\mathrm{XL}$ to initiate apoptosis. $\mathrm{BH} 3$ mimetics function to mimic sensitiser $\mathrm{BH}$-only proteins by binding to the $\mathrm{BH} 3$ domain of anti-apoptotics in a similar manner to endogenous $\mathrm{BH} 3-$ only proteins, displacing direct activators from anti-apoptotic proteins. The initial BH3-mimetic, the small molecule ABT-737, was designed to bind distinct regions within the $\mathrm{BH} 3$ domain using key residues based on Bak [31]. However, this design does not take into consideration the role the MOM plays in this interaction. Indeed, when bound to the MOM, Bcl-XL sequesters BH3-only proteins such as tBid, Bim and Puma in a much tighter interaction than when in the cytosol [32], [33]. Studying the ability of $\mathrm{BH} 3$ peptides to disrupt the interaction between cBid and $\mathrm{Bcl}-\mathrm{XL}$ in vitro using giant unilamellar vesicles showed that $\mathrm{Bim}$, Bad and Hrk are the most effective at disrupting this interaction. However, whilst the BH3-mimetics ABT-737 and ABT-263 were able to inhibit the formation of Bcl-XL-cBid complexes, they were inefficient in disrupting interactions that had already formed between $\mathrm{cBid}$ and $\mathrm{Bcl}-\mathrm{XL}$ in the membrane [34]. Thus, membrane interactions cause resistance to derepression by mimetics like ABT-737 as they fail to displace Bim and Puma from $\mathrm{Bcl}-\mathrm{XL}$. Intriguingly, sensitiser $\mathrm{BH} 3$-only proteins may not need to fully displace activators like Bim from Bcl-XL to activate Bax or Bak. A recent study has shown that 
Bad can allosterically activate cBid whilst both are in a complex with Bcl-XL [35]. In this model, Bcl-XL forms complexes at the MOM, binding both activator and sensitiser BH3-only proteins simultaneously. Sensitisers Bim or Bad can bind one $\mathrm{Bcl}-\mathrm{XL}$ in the complex, activating $\mathrm{cBid}$ bound to another $\mathrm{Bcl}-\mathrm{XL}$ in the complex, leading to the activation of Bax. Indeed, FRET two-hybrid assays confirmed that Bcl$\mathrm{XL}$ binds Bad as part of a complex with 2:1 stoichiometry [36]. This interaction is dependent on the presence of full-length proteins and the MOM, as a functional sensitiser $\mathrm{BH} 3$ domain in combination with the $\mathrm{Bcl}-\mathrm{XL}$ transmembrane targeting domain sequence is required.

This discovery has implications in the efficacy of BH3-mimetics, as ABT-263, a variant of $A B T-737$, was shown to only successfully activate Bax when cBid is fully displaced from $\mathrm{Bcl}-\mathrm{XL}$, and thus cannot activate Bax when $\mathrm{cBid}$ is still bound to $\mathrm{Bcl}-$ $X L$ [35]. This could suggest that mimetics are only effective at displacing weaker interacting sensitisers that can be more easily displaced from $\mathrm{Bcl}-\mathrm{XL}$, failing to activate a proportion of membrane-localized tBid. This raises the questions of which are the key residues involved in this interaction, and which membrane components are necessary for formation of these complexes. Intriguingly, resistance is beginning to emerge in patients over prolonged usage of $\mathrm{BH} 3$-mimetics such as Venetoclax [37]-[39]. These resistances can be mapped to specific mutations in key residues in the $\mathrm{BH} 3$ binding pocket of $\mathrm{Bcl}-2$ [38] which interestingly, inhibit only the binding of BH3-mimetics, but not Bcl-2 family proteins [40], [41]. Further study into the specific residues involved in this interaction could lead to improved BH3-mimetics efficacy in this regard.

\section{Direct activation versus direct inhibition of Bax and Bak}

In addition to their interactions with sensitiser BH3-only proteins, anti-apoptotic Bcl-2 proteins also directly inhibit Bax and Bak to prevent activation via $\mathrm{BH} 3-o n l y$ proteins. Interestingly, the significance of direct activators in the activation of Bax and Bak has been an area of debate. Whilst numerous in vitro studies have established a role for BH3-only proteins in activating Bax and Bak [10], [11], [42], some studies suggest that $\mathrm{BH} 3-o n l y$ protein interaction with Bax and Bak is not required for their activation, but rather inhibition of anti-apoptotic proteins is sufficient to induce MOMP. While Bax and Bak are not necessarily constitutively active and their inactive monomeric forms 
are present in the cytosol (Bax) and mitochondria (Bak) [43], [44], they can spontaneously activate in the absence of BH3-only proteins. For example, in HCT116 cells, inhibition of Bcl-XL and Mcl-1 can induce Bax-dependent apoptosis [25]. When these cells lack both activator and sensitizer BH3-only proteins, addition of full-length Bad can induce apoptosis when Mcl-1 is absent, or Noxa when Bcl-XL is absent [26]. Furthermore, in cells lacking all Bcl-2 proteins (Bcl-2 All KO cells), addition of Bax alone can induce apoptosis, which is inhibited when Bcl-XL is present. These studies suggest that neutralization of anti-apoptotic proteins is sufficient to allow the spontaneous membrane localization, activation and pore formation of Bax. These studies, of course, are not representative of the normal in vivo cell environment, but highlight the importance of targeting anti-apoptotic proteins in cells resistant to apoptosis induction. Indeed, systematic analysis of multiple cancer cell lines and primary patient samples has highlighted that their survival is dependent on specific anti-apoptotic $\mathrm{Bcl}-2$ proteins, with treatment with the appropriate BH3-mimetic sufficient to induce apoptosis [45]. Therefore, whilst BH3-only proteins are important in the activation of monomeric Bax and Bak, anti-apoptotic proteins are also crucial in inhibiting their spontaneous activation in the absence of $\mathrm{BH} 3$-only proteins.

Regulating the localization of Bax is therefore an important factor in the inhibition of apoptosis by anti-apoptotic $\mathrm{Bcl}-2$ proteins. As demonstrated in $\mathrm{Bcl}-2$ All KO cells, Bax is free to localize at the MOM, oligomerize and induce apoptosis without the need for other Bcl-2 family proteins [46]. Therefore, preventing Bax from localizing at the MOM should be an effective method to inhibit pore formation, at least where Bax is concerned. In the presence of other Bcl-2 family proteins, Bax localization is in constant equilibrium between the cytosol and the MOM [7]. The retrotranslocation of Bax from the MOM to cytosol by Bcl-XL is therefore key to preventing induction of apoptosis by shifting the balance of Bax in favour of its inactive, cytosolic form [6], [8]. Indeed, recent modelling analysis of this process demonstrates there is a fine balance in levels of $\mathrm{Bcl}-\mathrm{XL}$ retrotranslocating Bax from the MOM to inhibit apoptosis, and Bax overcoming this inhibition to induce apoptosis [47]. Only modest changes in Bcl-XL concentration can cause a "switch-like" transition to induce MOMP. This model does include a function for $\mathrm{BH} 3$-only proteins in this system, as their addition into the model demonstrates their ability potentiate Bax activation, partly by inhibiting Bcl-XL. 
Together these studies demonstrate the importance in inhibiting Bax localization at the MOM. Bak is also retrotranslocated from the MOM, but the rate of this is much slower than Bax, favouring a mainly mitochondrial localization [48]. Bak is kept in an inactive conformation at the membrane via inhibition by the mitochondrial porin VDAC2 [49]. What is unclear in these systems is how Bax, or indeed the inherently mitochondrial Bak, become active once there. Whilst spontaneous activation is possible, it seems $\mathrm{BH} 3-$ only proteins can act as effective inducers of this activation, catalysing the induction of the apoptotic response. An increasing level of complexity is emerging in the mechanism of this interaction when it is studied in more representative systems.

BH3-only proteins can activate Bax and Bak by binding their canonical groove to create BH3-exposed Bak/Bax [50], [51]. This conformational change causes a rearrangement of the $\mathrm{N}$ - and $\mathrm{C}$ terminus of Bax, separating a-helices 5 and 6 and inducing subsequent insertion of the $C$-terminus into the membrane [52]. Once inserted, homodimerization can occur via the a2-a5 "dimerization domain", with a dimer:dimer interface formed by the $\alpha 6-\alpha 9$ "piercing domain" of another molecule [50]. A similar mechanism has also been identified in Bak [53]. One of the most studied $\mathrm{BH} 3-\mathrm{Bax}$ interactions is with that of Bid. Bid is different to other $\mathrm{BH} 3$-only proteins in that it is intrinsically structured and requires cleavage via caspase 8 to activate its pro-apoptotic effect [54]. When cleaved by caspase 8 , Bid forms two fragments known as p7 and p15 (also known as tBid). Multiple studies suggest this cleavage occurs once Bid is at the MOM, rather than cleavage being the cause of this localisation [55], [56], and the $p 7$ fragment can remain associated with tBid after cleavage [56]. In order to activate Bax, Bid also requires interaction with the MOM even though Bid itself does not contain a $C$-terminal targeting sequence [57]. At the MOM, Bid undergoes conformational changes in a stepwise manner from a compact structure to a more elongated conformation to induce Bax activation. A recent study utilizing spin-label ESR and site-directed PEGylation shows at this stage, tBid undergoes a significant rearrangement of its $a 6-\alpha 8$ helices when in contact with the MOM, increasing accessibility of its $\mathrm{BH} 3$ domain a3 helix but maintaining overall structural integrity [58]. This is proposed to be a vital step in its interaction with Bax. Once associated with Bax, tBid then forms a more extended conformation via interaction with not only Bax and the MOM, but also Mtch2 [59]. tBid can then activate Bax via interaction with the $\mathrm{BH} 3$ binding groove [50]. Due to the transient 
nature of the Bid-Bax interaction, detecting Bid and Bax in complex at the MOM has proven difficult, however FCCS measurements in vitro using GUVs identifies a low affinity interaction between Bid and Bax [60] as well as FRET measurements in liposomes [35]. A recent study has also pinpointed specific residues of tBid:Bax interaction using FRET imaging, identifying residues between helices $\alpha 3$ and $\alpha 7$, and suggests that tBid helices $\alpha 5-\alpha 7$ might interact with the helix $\alpha 3$ of $\mathrm{Bax} \mathrm{BH} 3$ domain [56].

Delineating the mechanism by which Bax becomes activated by BH3-only proteins other than Bid has also been an area of interest over recent years. The $C$-terminus of Bim is not only important in regulating its mitochondrial localization and strengthening its interaction with Bcl-XL but is also important in regulating Bax activation[61]. Distinct residues within this sequence are involved in stabilizing the interaction with Bax BH3-binding groove (L129 and 1132), whilst having little effect on Bcl-XL interaction. PEGylation-based analysis of Bax binding groove was used to propose that $\mathrm{cBid}$ and Bim interact Bax via different surfaces, with Bim directly interacting with the trigger groove to release Bax a9 from its canonical groove, whereas $\mathrm{cBid}$ interacts with the canonical groove alongside mitochondrial lipids to activate Bax fully [62].

An alternate binding site has also been implicated in BH3-only mediated Bax activation other than its canonical $\mathrm{BH} 3$ binding groove. A second "rear groove" was identified encompassing helices $\alpha 1$ and $\alpha 6$ by which $\mathrm{BH} 3$-only proteins could also bind [63]. Introducing mutations in the $\alpha 1$ and $\alpha 6$ helices of Bax reduced $\mathrm{BH} 3$ binding and altered the conformation of Bax, inhibiting its ability to localize to the MOM [64]. These two sites could therefore have complementary functions, with the $\alpha 1$ and $\alpha 6$ site regulating the localization and integration of Bax into the MOM and the canonical site regulating dimerization. An alternate binding site has also been implicated in Bak activation. Bmf and Hrk, two BH3-only proteins whose specific apoptotic activating mechanisms are unclear, have recently been shown to not only directly bind at the hydrophobic groove of Bak, but can do so at an alternative site to the canonical site [65]. Bmf and Hrk can bind both the canonical a3/a4/a5 groove and the alternative a4/a6/a7 groove, with Bmf showing a mild preference for the non-canonical groove. These additional sites of regulation suggest that $\mathrm{BH} 3$-only proteins can aid in both the localization and activation of Bax and Bak to increase the potential of MOMP occurring. Indeed, analysis of two mutations in the Bax $\alpha 9$ helix - G179P, defective in 
membrane targeting, and T182I, able to target membranes but defective in membrane insertion - demonstrated that T182I mutant Bax was able to induce apoptosis similar to WT Bax, but G179P was not [66]. Thus, the residency of Bax at the MOM is crucial for apoptotic induction, which would be aided by binding of $\mathrm{BH}$ only proteins at a non-canonical site.

Together, these studies highlight that integration of both direct and indirect Bax/Bak activation models is most representative of the mechanism by which MOMP is initiated. Inhibition of Bax and Bak activation, either by retrotranslocation or direct binding at the MOM, is crucial in for preventing either autoactivation of Bax and Bak or direct activation by $\mathrm{BH} 3$-only proteins, with the $\mathrm{BH} 3$-only proteins catalysing this activation to induce conformation changes in Bax/Bak at the MOM to initiate pore formation.

\section{Formation of Bax/Bak pores in the MOM}

Whether directly or indirectly activated, the interplay between Bcl-2 family members ultimately culminates in Bax/Bak pore formation and permeabilization of the MOM [20]. The mechanism by which Bax and Bak oligomerise and the structure of subsequent pores has been an area of much interest in recent years. Previous studies reported Bax pores are composed of both protein and lipid molecules, which are relatively stable and can vary in size depending on protein concentration [67]. To form these pores, Bax/Bak undergo a conformational shift to open up its $\mathrm{N}$-terminus, freeing the a9 transmembrane helix for insertion into the MOM, with subsequent exposure of the $\mathrm{BH} 3$ domain. The details of how monomeric Bax and Bak then oligomerize are still under examination, but small molecule inhibitors which prevent dimerization also inhibit apoptosis induction in neurons, highlighting the need for higher order structures [68]. BH3-into-groove dimer formation between $\alpha 2-\alpha 5$ helices initiates the formation of pores, followed by an a9 helix from a neighboring Bax molecule forming another dimer interface within the membrane [69]. BH3-protein binding causes an opening between dimerization domain and piercing domain, separating the $a 5$ and $a 6$ pore-forming hairpin [50], [70]. Analyzing Bax oligomers at a single molecule level showed that assembly of monomeric to multiple coexisting Bax oligomers based on dimer units occurs rapidly once inserted into the MOM [22]. Dimers of Bak have also been identified to form in a similar manner [53]. Examination 
of Bak oligomerization using chemical crosslinking and double electron electron resonance-derived measurements between different areas within the Bak structure showed multiple interacting interfaces between two Bak homodimers, namely the $\alpha 3 / a 5, a 6 / \alpha 6$ and $a 9 / \alpha 9$ [71]. Crystal structure analysis of Bak dimers showed the significant role of lipids in this interaction, with the two acyl chains of each headgroup associating with two different Bak dimers, providing flexibility in their association [72].

Bax and Bak are believed to form toroidal pores, with the insertion of Bax generating membrane tension which is reduced by the opening of a pore, with subsequent lipid rearrangements around the pore, as recently reviewed in [73]. Thanks to the use of advanced microscopy techniques, the architecture of Bax pores has been visualized in detail. Using single-molecule localization and STED super-resolution microscopy, Bax can be seen forming ring and arc structures capable of permeabilizing the membrane [74], [75]. Not only most rings, but also a fraction of the arcs are associated with membrane pores, indicating that complete coverage of the pore edge is not necessary for stabilizing the open pore state. The exact role of linear structures is still unclear, but they could potentially represent stages leading to full pore formation or kinetically trapped intermediates.

The new information from the use of novel model systems and imaging techniques has therefore added to the complexity of the regulation and mechanism of MOMP induction. In this scenario, the role of the membrane environment in the regulation of both the activation and activity of the executioners Bax and Bak has now been recognized. Understanding the roles of mitochondrial proteins outside of the $\mathrm{Bcl}-2$ family and how they contribute to the mechanism regulating Bax and Bak activation and pore formation is therefore crucial in understanding the final moments of apoptotic regulation, and the roles of various structures of oligomeric Bax and Bak. Once these are taken into consideration, we will be closer to a fuller picture of this key process.

\section{MOMP regulation by other mitochondrial components}

\section{Mitochondrial dynamics in apoptosis regulation}

Mitochondria are highly dynamic structures, which form interconnected tubules that continuously undergo fusion and fission by the action of large GTPase dynamin-like 
proteins resulting in shuffling of mitochondrial context within the network in living cells. This dynamic transformation also regulates mitochondria intracellular distribution and their movement along the cytoskeleton. Fusion of the MOM is mediated by mitofusin 1 (Mfn1), mitofusin 2 (Mfn2), while optic atrophy 1 protein (OPA1) mediates the fusion of the mitochondrial inner membrane (MIM). On the other hand, mitochondrial fission is regulated mainly by dynamin-related protein 1 (Drp1). Mitochondrial dynamics have been also associated with cell death and numerous studies have shown a connection between Bcl-2 family members (Bax, Bak, Bcl-2 and $\mathrm{Bcl}-\mathrm{xL}$ ) and proteins involved in mitochondrial morphogenesis (Mfn1, Mfn2, and Drp1) [76]-[78]. During the execution of the apoptotic program, mitochondria undergo extensive fragmentation regulated by fusion/fission proteins. SUMOylation of Drp1, stabilizes Drp1 on mitochondrial membrane and colocalizes with Mfn2 and Bax. SUMOylated Drp1 functionally stabilizes ER/mitochondrial contact sites and creating a platform for mitochondrial constriction, calcium flux, cristae remodeling, and cytochrome $c$ release [79]. Mitochondrial-anchored protein/SUMO ligase (MAPL)-mediated SUMOylation of Drp1 has also been shown to stabilizes its oligomeric form on the MOM resulting in increased mitochondrial fission during apoptosis (Fig. 2d). Notably, selective inhibition of Drp1 oligomerization resulting in elongated mitochondrial network and apoptosis inhibition [80], [81].

Remodeling of cristae architecture plays a role in cytochrome $c$ release during MOMP and apoptosis. OPA1 regulates MIM fusion, therefore maintaining cristae junction formation and size. OPA1 oligomers keep junctions narrow, whereas disassembly of OPA1 oligomers widen the junction [82]-[84]. Bax and Bak facilitate Mfn2 oligomerization and activity in healthy cells, while during apoptosis, Bak dissociates from Mfn2 and associates with Mfn1 [77], [85]. Conversely, Mfn1 phosphorylation facilitates Bak oligomerization in response to apoptotic stimuli. This is regulated by phosphorylation of Mfn1, which inhibits its pro-fusion function and triggers its association with Bak, which favours mitochondria fragmentation, Bak oligomerization and therefore cytochrome $c$ release and cell death [86].

However, much of the research so far connecting mitochondrial dynamics with apoptosis suffers from the difficulty to disentangle whether the mitochondria fussion and fission machinery impacts directly on the apoptosis pathway, or whether it has an indirect effect via alterations in the functional state of mitochondria, which eventually 
affect apoptosis sensitivity. As a result, the structural basis of these connections, the molecular mechanisms involved and the functional consequences remain largely unclear.

\section{Mitochondrial recruitment of BCL-2 family proteins}

The process of MOMP orchestrated by $\mathrm{Bcl}-2$ family proteins requires an activator to trigger the activation of effector proteins. This process is also regulated by mitochondrial proteins known to facilitate the recruitment of $\mathrm{Bcl}-2$ family proteins at the MOM [15], [87]. Under apoptotic stress, cytosolic Bid is post-translationally cleaved by caspase-8 to produce truncated Bid (tBid). tBid binds the MOM and undergoes a conformational change to further activate Bax. tBid binding to the MOM is facilitated by the MOM protein Mtch2, which accelerates the re-localization of tBid at the mitochondria and regulates cell apoptosis [59], [88]. Mtch2 promotes tBid binding to the MOM by enhancing a conformational change of tBid that leads to deeper membrane insertion and is required for its Bax activation capability (A. Shamas-Din et al. 2014; Aisha Shamas-Din et al. 2013).

Voltage-dependent anion channel 2 (VDAC2) is another example of a mitochondrial protein which interacts with Bax and Bak, thereby regulating MOMP and cell death [89]-[91]. VDAC2 has been shown to act as a platform for Bax at the MOM and to inhibit its retrotranslocation back the cytosol (Fig. 2b) [6], [15]. In addition, Bax localized to other cell compartments in the absence of VDAC2 [15], [48]. Recently, genetic ablation of VDAC2 has been shown to have a significant impairment of the mitochondrial integration of Bak and Bax, and clarified that efficient Bax-mediated apoptosis depends on VDAC2 [92]. VDAC2 interactions with Bak, Bax and tBid have been examined using atomic-level structure-based modeling to estimated their binding affinities for VDAC2, which led to the hypothesis that tBid displaces Bak from the VDAC2/Bak complex followed by the formation of the VDAC2/tBid complex which further recruits Bax to the MOM upon apoptosis induction [93]. In a recent study, using a photoactivatable ceramide probe combined with computer simulations and functional studies identified VDAC2 as a critical effector of ceramide-induced mitochondrial apoptosis [94]. The authors proposed that ceramide binding to VDAC2 may commit cells to death by blocking Bax retrotranslocation. Ceramide binding to VDACs was also suggested to influence interactions with other proteins, as the 
ceramide binding site at VDACs is also critical for their association of with hexokinase I [95].

Mitochondrial residing Hexokinases (HKs) are also known to interfere with members of the Bcl-2 family and to regulate Bax-mediated mitochondrial membrane injury [96], [97]. Overexpression of HKs and their association with VDACs are typical features of hyperglycolytic cancer cells [98], [99]. When bound to VDACs, HKs enable an effective coupling between oxidative phosphorylation (OXPHOS) and glycolysis by capturing ATP released from mitochondria for production of glucose-6-phosphate, the rate limiting step in the glycolytic cascade. Consequently, VDAC-HK interactions have drawn considerable interest as a potential pharmaceutical target for the development of novel anti-cancer agents [100]. A recent study has shown that high glucose may induce apoptosis via downregulation of $\mathrm{HK} 2$, leading to decreased interactions of HK2 with VDACs and increased VDACs-Bax association, with the subsequent increased release of cytochrome $c$ [101]. These observations suggest that Bax and HKs possibly share a common binding site on VDACs at the MOM [95], [96], [101].

\section{Regulation of Bax and Bak activity by non-Bcl-2 proteins}

Bax and Bak are also regulated independently of Bcl-2 proteins. E3 ubiquitin ligase, Parkin, and its upstream regulator PTEN-induced Kinase 1 (PINK1) are involved in the removal of damaged mitochondria through a selective form of autophagy. In cooperation with OPA1 and sorting nexin 9 (Snx9)-mediated selective packaging of mitochondrial proteins, Parkin plays an important role in mitochondrial quality control [102]. Mitochondrial insults such as membrane depolarization, or accumulation of misfolded mitochondrial proteins, stabilizes PINK1 on the MOM with subsequent homodimerization, which promotes activation of its kinase activity and binding to Parkin and ubiquitin. PINK1/Parkin ubiquitinates a range of mitochondrial substrates and triggers the clearance of damaged mitochondria [103]. Parkin-mediated ubiquitination has been shown to modulate cellular metabolism, mitochondrial dynamics, cell cycle and apoptosis [104], [105]. Recently, Parkin has been suggested to also ubiquitylate Bax, thereby targeting cytosolic Bax for proteasomal degradation [16], [106]. Parkin-dependent ubiquitination of endogenous Bax explains the antiapoptotic effects of Parkin. Moreover, the $\mathrm{BH} 3$ domain of Bax was found to be critical 
for its recognition by Parkin [107]. Parkin is also reported to ubiquitinate Bak at a conserved lysine in its hydrophobic groove, which is crucial for Bak activation by BH3-only proteins and for its homo-dimerization during apoptosis [53], [108], [109].

$\mathrm{Bcl}-2$ ovarian killer (BOK) is a less understood member of the Bcl-2 family classified as a Bax-like protein and as an effector of MOMP [110], [111]. Bok has been shown to trigger membrane permeabilization and apoptosis even in the absence of Bax and Bak [112], [113]. Unlike Bax and Bak, Bok is constitutively active, does not require activation by $\mathrm{BH} 3$-only proteins and is unresponsive to the antagonistic effects of the anti-apoptotic Bcl-2 proteins [111]. The molecular mechanism regulating BOK function is poorly understood and restricted to selective proteasomal turnover regulated by the endoplasmic reticulum (ER)-associated degradation (ERAD) pathway [112], [114]. In a recent study, BOK was shown to locate to the mitochondrial associated membrane (MAM), and demonstrated that their interaction with the MAM protein inositol 1,4,5-trisphosophate receptor (IP3R) is critical for regulating $\mathrm{Ca} 2+$ transport from the $\mathrm{ER}$ to the mitochondria, for ER-mitochondrial interaction sites and for apoptosis [115], [116].

\section{Alterations in mitochondrial lipids during apoptosis}

It is widely accepted that Bax/Bak oligomers and membrane lipids are key components of the apoptotic pore, which has a proteolipidic nature [74], [117]. The mitochondrial membrane lipid environment was found to be an important factor for the activation of Bax and thereby may affect MOMP. Concretely, the mitochondrialspecific lipid cardiolipin $(\mathrm{CL})$ has been reported to become transiently exposed on the mitochondrial surface after apoptosis induction and thought to promote Bax recruitment and MOMP [17], [118]. The presence of $C L$ in the membrane has also been reported essential for tBID recruitment and BAX insertion, which support a direct role of CL in Bax-mediated apoptosis [119]-[122].

Since Drp1 locates to Mitochondria-ER contact sites (MERCs) and Drp1 colocalizes with Bax and Bak in apoptosis, it is likely that MERCs and Bax/Bak foci are functionally connected [79], [80], [123], [124]. MERCs is a major source for the exchange of lipids between ER and mitochondria and demonstrated as equally important player in mitochondrial apoptosis. Recently, this contact site has been shown to involved in lipids translocation to mitochondria to induce MOMP and 
apoptosis. Green and coworkers proposed that ceramide derived from sphingomyelin in the MERCs is transferred to mitochondria, where sphingolipid intermediate products, sphingosine-1-PO4 and hexadecenal, promote Bak and Bax activation, respectively, leading to MOMP and cytochrome $c$ release [125]. Increased mitochondrial ceramide has been also shown to enhance Bax translocation to the mitochondria and Bax-dependent apoptosis [126].

Besides the ER, mitochondria form stable contacts with other organelles, such as endosomes/lysosomes (EL). The physical coupling of these organelles has been proposed to lead to the transfer of cholesterol from endosomes to mitochondria [127]. During oxidative stress, an increase association has been shown between Rab5positive endosomes and mitochondria (Hsu et al. 2018). This phenomenon has been recapitulated upon apoptotic induction and accumulation of Rab5-positive endosomes have been observed at the proximity of Bax clusters regulating the release of cytochrome $c$ and SMAC into the cytosol (Fig. 2c). Remarkably, depleting or interfering with Rab5 activation and recruitment to apoptotic mitochondria impaired mitochondrial pore formation and the subsequent release of IMS proteins [18]. This study suggested that EL targeting to mitochondria during apoptosis altered the lipid environment at the MOM, specifically via the transfer of cholesterol to mitochondria, which would favor BAX accumulation and conformational changes.

Lysosomes are essential in regulating lipid metabolism, and also affect endocytosis and apoptosis. Lysosomal V-ATPase, a proton pump necessary for lysosomal acidification, has been shown to induce mitochondria-driven apoptosis in different cancer cells [128], [129]. However, the detailed mechanisms leading to mitochondrial apoptosis induction remain unknown.

\section{Conclusions and future perspective}

$\mathrm{Bcl}-2$ family members including the $\mathrm{BH} 3$-only proteins and the anti-apoptotic $\mathrm{Bcl}-2$ proteins control the activation and activity of Bax and Bak, which determines the fate of the cell. Current understanding in this field has culminated in the development of BH3-mimetics as an effective way to sensitise cells to mitochondrial apoptosis. These have shown great success in clinical settings, such as Venetoclax for the treatment of chronic lymphocytic leukaemia as a single agent [39], or as a combination treatment 
for acute myeloid leukaemia [130]. The potential for use of BH3-mimetics to treat other types of cancer and, in particular, solid tumours, is an area of great promise. As reviewed here, advances in our understanding of the binding mechanism between anti- and pro-apoptotic Bcl-2 proteins may aid in the design of $\mathrm{BH} 3$ mimetics with improved clinical characteristics.

Furthermore, mounting evidence continues to highlight the importance of the membrane environment, mitochondrial lipid composition and mitochondrial dynamics to regulate Bax and Bak apoptotic function. Beyond the interplay between Bcl-2 family proteins, mitochondrial components such as VDACs, HKs and Mtch2 and lipids also play a significant role in Bax/Bak regulation. Additional factors including mitochondrial dynamics and membrane contact sites also regulate this process. How these many players could be manipulated in new ways to modulate apoptosis opens new and exciting opportunities that could be exploited to kill therapy-resistant cancer cells.

\section{Figure legend}

Figure 1. Bax and Bak activation models. (A) Direct activation model. Bax and Bak are activated by direct binding of the $\mathrm{BH} 3$ domain of activator $\mathrm{BH} 3$-only proteins (denoted "A") with the Bax/Bak hydrophobic groove. This causes a conformational change in Bax/Bak initiating oligomerisation, MOM pore formation and cytochrome $\mathrm{C}$ release. This activation is inhibited by sequestration of the direct activators by the anti-apoptotic proteins (denoted "Anti"). Direct activators can be displaced from antiapoptotic proteins by the sensitiser BH3-only proteins (denoted "S"). (B) Indirect activation model. Bax and Bak are constitutively active and targeted to the MOM, but are inhibited by anti-apoptotic Bcl-2 proteins. BH3-only proteins are not required for direct Bax/Bak activation, but rather inhibit anti-apoptotic proteins, freeing Bax/Bak to oligomerise. In this model there is no distinction between direct activator and sensitiser BH3-only proteins. (C) Embedded together model. The activation of Bax and Bak is dependent on interactions with the MOM which can be inhibited by antiapoptotic proteins retrotranslocating Bax/Bak to the cytosol. Direct activator BH3-only proteins interact with Bax/Bak at the MOM, inducing a conformational change to initiate Bax/Bak oligomerisation. Direct activators are inhibited by anti-apoptotic proteins, which themselves can be sequestered by sensitiser BH3-only proteins. Interactions with the MOM regulate the displacement of direct activators from the anti-apoptotic proteins.

Figure 2. Schematic representation of factors regulating Bax/Bak-mediated MOMP. (A) Activated Bax self-assembles into a cytochrome $c$-conducting pore. Due to a lower rate of retrotranslocation BAK is mostly MOM-bound. (B) VDACs mediated 
recruitment and assembly of Bax at the MOM. VDACs serve as platform for Bax on the surface of mitochondria and promoting cytochrome $c$ release via MOMP. (C) Endolysosomes recruitment to the mitochondria during apoptosis facilitates BAX clustering and MOMP. (D) SUMOylated Drp1 at the ER/mitochondria interface during apoptosis. SUMOylated Drp1 functionally stabilizes an ER/mitochondrial contact site and facilitates Bax-mediated cytochrome $c$ release.

\section{Acknowledgements}

This manuscript was supported by the European Research Council (ERC) under the European Union's Horizon 2020 research and innovation program (Grant agreement No. 817758).

\section{Author contributions}

Ana J. Garcia-Saez conceptualized this manuscript with an equal contribution from Shashank Dadsena and Louise E. King.

\section{Declaration of competing interest}

The authors declare no competing interests.

\section{References}

[1] N. N. Danial and S. J. Korsmeyer, "Cell Death: Critical Control Points," Cell, vol. 116, no. 2. 2004, doi: 10.1016/S0092-8674(04)00046-7.

[2] H. Kalkavan and D. R. Green, "MOMP, cell suicide as a BCL-2 family business," Cell Death Differ., vol. 25, no. 1, pp. 46-55, 2018, doi: 10.1038/cdd.2017.179.

[3] R. J. Youle and A. Strasser, "The BCL-2 protein family: Opposing activities that mediate cell death," Nature Reviews Molecular Cell Biology, vol. 9, no. 1. Nature Publishing Group, pp. 47-59, Jan-2008, doi: 10.1038/nrm2308.

[4] J. E. Chipuk, T. Moldoveanu, F. Llambi, M. J. Parsons, and D. R. Green, "The BCL-2 Family Reunion,” Molecular Cell. 2010, doi: 10.1016/j.molcel.2010.01.025.

[5] D. Westphal, R. M. Kluck, and G. Dewson, "Building blocks of the apoptotic pore: How Bax and Bak are activated and oligomerize during apoptosis," Cell Death and Differentiation, vol. 21, no. 2. 2014, doi: 10.1038/cdd.2013.139.

[6] F. Edlich et al., "Bcl-xL retrotranslocates Bax from the mitochondria into the cytosol," Cell, vol. 145, no. 1, pp. 104-116, 2011, doi: 
10.1016/j.cell.2011.02.034.

[7] B. Schellenberg et al., "Bax Exists in a Dynamic Equilibrium between the Cytosol and Mitochondria to Control Apoptotic Priming," Mol. Cell, 2013, doi: 10.1016/j.molcel.2012.12.022.

[8] F. Todt, Z. Cakir, F. Reichenbach, R. J. Youle, and F. Edlich, "The C-terminal helix of Bcl-x $L$ mediates Bax retrotranslocation from the mitochondria," Cell Death Differ., 2013, doi: 10.1038/cdd.2012.131.

[9] H. Kim et al., "Stepwise Activation of BAX and BAK by tBID, BIM, and PUMA Initiates Mitochondrial Apoptosis," Mol. Cell, 2009, doi: 10.1016/j.molcel.2009.09.030.

[10] A. Letai, M. C. Bassik, L. D. Walensky, M. D. Sorcinelli, S. Weiler, and S. J. Korsmeyer, "Distinct $\mathrm{BH} 3$ domains either sensitize or activate mitochondrial apoptosis, serving as prototype cancer therapeutics," Cancer Cell, 2002, doi: 10.1016/S1535-6108(02)00127-7.

[11] T. Kuwana et al., "BH3 domains of $\mathrm{BH} 3-o n l y$ proteins differentially regulate Bax-mediated mitochondrial membrane permeabilization both directly and indirectly," Mol. Cell, vol. 17, no. 4, 2005, doi: 10.1016/j.molcel.2005.02.003.

[12] M. C. Wei et al., "tBID, a membrane-targeted death ligand, oligomerizes BAK to release cytochrome c," Genes Dev., vol. 14, no. 16, 2000, doi: 10.1101/gad.14.16.2060.

[13] S. N. Willis et al., "Apoptosis initiated when $\mathrm{BH} 3$ ligands engage multiple Bcl-2 homologs, not Bax or Bak," Science (80-. )., 2007, doi: 10.1126/science.1133289.

[14] A. Shamas-Din et al., "TBid undergoes multiple conformational changes at the membrane required for bax activation," J. Biol. Chem., 2013, doi: 10.1074/jbc.M113.482109.

[15] J. Lauterwasser et al., "The porin VDAC2 is the mitochondrial platform for Bax retrotranslocation," Sci. Rep., 2016, doi: 10.1038/srep32994.

[16] B. N. Johnson, A. K. Berger, G. P. Cortese, and M. J. LaVoie, "The ubiquitin E3 ligase parkin regulates the proapoptotic function of Bax," Proc. Natl. Acad. Sci. U. S. A., vol. 109, no. 16, 2012, doi: 10.1073/pnas.1113248109.

[17] T. Kuwana et al., "Bid, Bax, and lipids cooperate to form supramolecular openings in the outer mitochondrial membrane," Cell, 2002, doi: 10.1016/S0092-8674(02)01036-X. 
[18] T. Sen Wang, I. Coppens, A. Saorin, N. R. Brady, and A. Hamacher-Brady, "Endolysosomal Targeting of Mitochondria Is Integral to BAX-Mediated Mitochondrial Permeabilization during Apoptosis Signaling.," Dev. Cell, vol. 53, no. 6, pp. 627-645.e7, Jun. 2020, doi: 10.1016/j.devcel.2020.05.014.

[19] B. Leber, J. Lin, and D. W. Andrews, "Embedded together: The life and death consequences of interaction of the Bcl-2 family with membranes," Apoptosis. 2007, doi: 10.1007/s10495-007-0746-4.

[20] J. F. Lovell et al., "Membrane Binding by tBid Initiates an Ordered Series of Events Culminating in Membrane Permeabilization by Bax," Cell, 2008, doi: 10.1016/j.cell.2008.11.010.

[21] S. Bleicken, C. Wagner, and A. J. García-Sáez, "Mechanistic differences in the membrane activity of bax and Bcl-xL correlate with their opposing roles in apoptosis," Biophys. J., 2013, doi: 10.1016/j.bpj.2012.12.010.

[22] Y. Subburaj et al., "Bax monomers form dimer units in the membrane that further self-assemble into multiple oligomeric species," Nat. Commun., 2015, doi: $10.1038 /$ ncomms 9042 .

[23] M. Certo et al., "Mitochondria primed by death signals determine cellular addiction to antiapoptotic BCL-2 family members," Cancer Cell, vol. 9, no. 5, 2006, doi: 10.1016/j.ccr.2006.03.027.

[24] J. Kale, E. J. Osterlund, and D. W. Andrews, "BCL-2 family proteins: Changing partners in the dance towards death," Cell Death and Differentiation, vol. 25, no. 1. 2018, doi: 10.1038/cdd.2017.186.

[25] G. Greaves et al., "BH3-only proteins are dispensable for apoptosis induced by pharmacological inhibition of both MCL-1 and BCL-XL," Cell Death Differ., vol. 26, no. 6, 2019, doi: 10.1038/s41418-018-0183-7.

[26] K. Huang et al., "BH3-only proteins target BCL-xL/MCL-1, not BAX/BAK, to initiate apoptosis," Cell Res., vol. 29, no. 11, pp. 942-952, Nov. 2019, doi: 10.1038/s41422-019-0231-y.

[27] A. M. Petros et al., "Rationale for Bcl-X L /Bad peptide complex formation from structure, mutagenesis, and biophysical studies ," Protein Sci., vol. 9, no. 12, 2000, doi: 10.1110/ps.9.12.2528.

[28] A. Aranovich et al., "Differences in the Mechanisms of Proapoptotic BH3 Proteins Binding to Bcl-XL and Bcl-2 Quantified in Live MCF-7 Cells," Mol. Cell, vol. 45, no. 6, 2012, doi: 10.1016/j.molcel.2012.01.030. 
[29] Q. Liu, E. J. Oesterlund, X. Chi, J. Pogmore, B. Leber, and D. W. Andrews, "Bim escapes displacement by BH3- mimetic anti-cancer drugs by double-bolt locking both Bcl-XL and Bcl-2," Elife, vol. 8, 2019, doi: 10.7554/eLife.37689.

[30] C. Hockings et al., "Bid chimeras indicate that most BH3-only proteins can directly activate Bak and Bax, and show no preference for Bak versus Bax," Cell Death Dis., vol. 6, no. 4, 2015, doi: 10.1038/cddis.2015.105.

[31] T. Oltersdorf et al., "An inhibitor of Bcl-2 family proteins induces regression of solid tumours," Nature, vol. 435, no. 7042, 2005, doi: 10.1038/nature03579.

[32] J. Pécot et al., "Tight Sequestration of BH3 Proteins by BCL-xL at Subcellular Membranes Contributes to Apoptotic Resistance," Cell Rep., vol. 17, no. 12, 2016, doi: 10.1016/j.celrep.2016.11.064.

[33] A. J. García-Sáez, J. Ries, M. Orzáez, E. Pérez-Payà, and P. Schwille, "Membrane promotes tBID interaction with BCL XL," Nat. Struct. Mol. Biol., vol. 16, no. 11, pp. 1178-1185, Nov. 2009, doi: 10.1038/nsmb.1671.

[34] K. K. Das, R. Shalaby, and A. J. García-Sáez, "Determinants of BH3 Sequence Specificity for the Disruption of Bcl-xL/cBid Complexes in Membranes," ACS Chem. Biol., vol. 12, no. 4, 2017, doi: 10.1021/acschembio.6b01084.

[35] C. Bogner et al., "Allosteric Regulation of $\mathrm{BH} 3$ Proteins in $\mathrm{Bcl}-\mathrm{xL}$ Complexes Enables Switch-like Activation of Bax," Mol. Cell, vol. 77, no. 4, 2020, doi: 10.1016/j.molcel.2019.12.025.

[36] W. Qu et al., "Gaussian FRET two-hybrid assays for determining the stoichiometry of hetero-oligomeric complexes in single living cells," Biochem. Biophys. Res. Commun., vol. 512, no. 3, 2019, doi: 10.1016/j.bbrc.2019.03.089.

[37] A. Gilmore and L. King, "Emerging approaches to target mitochondrial apoptosis in cancer cells," F1000Research, vol. 8, 2019, doi: 10.12688/f1000research.18872.1.

[38] E. Tausch et al., "Venetoclax resistance and acquired BCL2 mutations in chronic lymphocytic leukemia," Haematologica, vol. 104, no. 9. 2019, doi: 10.3324/haematol.2019.222588.

[39] S. Stilgenbauer et al., "Venetoclax for patients with chronic lymphocytic leukemia with 17p deletion: Results from the full population of a phase ii pivotal trial," J. Clin. Oncol., vol. 36, no. 19, 2018, doi: 10.1200/JCO.2017.76.6840.

[40] R. W. Birkinshaw et al., "Structures of BCL-2 in complex with venetoclax reveal 
the molecular basis of resistance mutations," Nat. Commun., vol. 10, no. 1, 2019, doi: 10.1038/s41467-019-10363-1.

[41] P. Blombery et al., "Characterization of a novel venetoclax resistance mutation (BCL2 Phe104lle) observed in follicular lymphoma," British Journal of Haematology, vol. 186, no. 6. 2019, doi: 10.1111/bjh.16069.

[42] H. C. Chen et al., "An interconnected hierarchical model of cell death regulation by the BCL-2 family," Nat. Cell Biol., 2015, doi: 10.1038/ncb3236.

[43] M. Suzuki, R. J. Youle, and N. Tjandra, "Structure of Bax: coregulation of dimer formation and intracellular localization.," Cell, vol. 103, no. 4, pp. 645-54, Nov. 2000, doi: 10.1016/s0092-8674(00)00167-7.

[44] S. Ma et al., "Assembly of the bak apoptotic pore: A critical role for the bak proteina6 helix in the multimerization of homodimers during apoptosis," J. Biol. Chem., vol. 288, no. 36, pp. 26027-26038, Sep. 2013, doi: 10.1074/jbc.M113.490094.

[45] R. S. Soderquist et al., "Systematic mapping of BCL-2 gene dependencies in cancer reveals molecular determinants of BH3 mimetic sensitivity," Nat. Commun., vol. 9, no. 1, 2018, doi: 10.1038/s41467-018-05815-z.

[46] K. L. O'neill, K. Huang, J. Zhang, Y. Chen, and X. Luo, "Inactivation of prosurvival Bcl-2 proteins activates Bax/Bak through the outer mitochondrial membrane," Genes Dev., 2016, doi: 10.1101/gad.276725.115.

[47] A. Hantusch, K. K. Das, A. J. Garciá-Saéz, T. Brunner, and M. Rehm, "Bax retrotranslocation potentiates Bcl-xL's antiapoptotic activity and is essential for switch-like transitions between MOMP competency and resistance," Cell Death Dis., 2018, doi: 10.1038/s41419-018-0464-6.

[48] F. Todt et al., "Differential retrotranslocation of mitochondrial Bax and Bak," EMBO J., vol. 34, no. 1, pp. 67-80, Jan. 2015, doi: 10.15252/embj.201488806.

[49] E. H. Y. Y. Cheng, T. V. Sheiko, J. K. Fisher, W. J. Craigen, and S. J. Korsmeyer, "VDAC2 inhibits BAK activation and mitochondrial apoptosis.," Science (80-. )., vol. 301, no. July, pp. 513-7, 2003, doi: 10.1126/science.1083995.

[50] P. E. Czabotar et al., "Bax crystal structures reveal how $\mathrm{BH} 3$ domains activate Bax and nucleate its oligomerization to induce apoptosis," Cell, 2013, doi: 10.1016/j.cell.2012.12.031.

[51] A. Y. Robin et al., "Crystal structure of Bax bound to the BH3 peptide of Bim 
identifies important contacts for interaction," Cell Death Dis., vol. 6, no. 7, 2015, doi: 10.1038/cddis.2015.141.

[52] S. Bleicken, T. E. Assafa, C. Stegmueller, A. Wittig, A. J. Garcia-Saez, and E. Bordignon, "Topology of active, membrane-embedded Bax in the context of a toroidal pore," Cell Death and Differentiation, vol. 25, no. 10. Nature Publishing Group, pp. 1717-1731, Nov-2018, doi: 10.1038/s41418-018-0184-6.

[53] J. M. Brouwer et al., "Bak Core and Latch Domains Separate during Activation, and Freed Core Domains Form Symmetric Homodimers," Mol. Cell, vol. 55, no. 6, 2014, doi: 10.1016/j.molcel.2014.07.016.

[54] J. J. Chou, H. Li, G. S. Salvesen, J. Yuan, and G. Wagner, "Solution structure of BID, an intracellular amplifier of apoptotic signaling," Cell, vol. 96, no. 5, 1999, doi: 10.1016/S0092-8674(00)80572-3.

[55] Z. T. Schug, F. Gonzalvez, R. H. Houtkooper, F. M. Vaz, and E. Gottlieb, "BID is cleaved by caspase- 8 within a native complex on the mitochondrial membrane," Cell Death Differ., vol. 18, no. 3, 2011, doi: 10.1038/cdd.2010.135.

[56] R. F. Gahl, P. Dwivedi, and N. Tjandra, "Bcl-2 proteins bid and bax form a network to permeabilize the mitochondria at the onset of apoptosis," Cell Death Dis., vol. 7, no. 10, 2016, doi: 10.1038/cddis.2016.320.

[57] K. Huang et al., "Cleavage by caspase 8 and mitochondrial membrane association activate the BH3-only protein bid during TRAIL-induced apoptosis," J. Biol. Chem., vol. 291, no. 22, 2016, doi: 10.1074/jbc.M115.711051.

[58] C. L. Hung, H. H. Chang, S. W. Lee, and Y. W. Chiang, "Stepwise activation of the pro-apoptotic protein Bid at mitochondrial membranes," Cell Death Differ., 2021, doi: 10.1038/s41418-020-00716-5.

[59] C. Katz, Y. Zaltsman-Amir, Y. Mostizky, N. Kollet, A. Gross, and A. Friedler, "Molecular basis of the interaction between proapoptotic truncated BID (tBID) protein and mitochondrial carrier homologue $2(\mathrm{MTCH} 2)$ protein: Key players in mitochondrial death pathway," J. Biol. Chem., 2012, doi: 10.1074/jbc.M111.328377.

[60] S. Bleicken, A. Hantusch, K. K. Das, T. Frickey, and A. J. Garcia-Saez, "Quantitative interactome of a membrane Bcl-2 network identifies a hierarchy of complexes for apoptosis regulation," Nat. Commun., vol. 8, no. 1, pp. 1-15, Dec. 2017, doi: 10.1038/s41467-017-00086-6.

[61] X. Chi et al., "The carboxyl-terminal sequence of bim enables bax activation 
and killing of unprimed cells," Elife, vol. 9, 2020, doi: 10.7554/eLife.44525.

[62] Y. J. Lan, Y. T. Wang, C. L. Hung, and Y. W. Chiang, "PEGylation-based strategy to identify pathways involved in the activation of apoptotic BAX protein," Biochim. Biophys. Acta - Gen. Subj., vol. 1864, no. 4, 2020, doi: 10.1016/j.bbagen.2020.129541.

[63] E. Gavathiotis et al., "BAX activation is initiated at a novel interaction site," Nature, vol. 455, no. 7216, 2008, doi: 10.1038/nature07396.

[64] M. A. Dengler et al., "BAX Activation: Mutations Near Its Proposed Noncanonical BH3 Binding Site Reveal Allosteric Changes Controlling Mitochondrial Association," Cell Rep., vol. 27, no. 2, 2019, doi: 10.1016/j.celrep.2019.03.040.

[65] K. Ye et al., "Characterization of an alternative BAK-binding site for $\mathrm{BH} 3$ peptides," Nat. Commun., vol. 11, no. 1, pp. 1-14, Dec. 2020, doi: 10.1038/s41467-020-17074-y.

[66] T. Kuwana et al., "Mitochondrial residence of the apoptosis inducer BAX is more important than BAX oligomerization in promoting membrane permeabilization," J. Biol. Chem., p. jbc.RA119.011635, Jan. 2020, doi: 10.1074/jbc.ra119.011635.

[67] S. Bleicken, O. Landeta, A. Landajuela, G. Basañez, and A. J. García-Sáez, "Proapoptotic Bax and Bak proteins form stable protein-permeable pores of tunable size," J. Biol. Chem., 2013, doi: 10.1074/jbc.M113.512087.

[68] X. Niu et al., "A Small-Molecule Inhibitor of Bax and Bak Oligomerization Prevents Genotoxic Cell Death and Promotes Neuroprotection," Cell Chem. Biol., vol. 24, no. 4, 2017, doi: 10.1016/j.chembiol.2017.03.011.

[69] Z. Zhang et al., "BH3-in-groove dimerization initiates and helix 9 dimerization expands Bax pore assembly in membranes," EMBO J., vol. 35, no. 2, pp. 208236, Jan. 2016, doi: 10.15252/embj.201591552.

[70] S. Bleicken, G. Jeschke, C. Stegmueller, R. Salvador-Gallego, A. J. GarcíaSáez, and E. Bordignon, "Structural Model of Active Bax at the Membrane," Mol. Cell, 2014, doi: 10.1016/j.molcel.2014.09.022.

[71] T. Mandal et al., "Assembly of Bak homodimers into higher order homooligomers in the mitochondrial apoptotic pore," Sci. Rep., 2016, doi: 10.1038/srep30763.

[72] A. D. Cowan et al., "BAK core dimers bind lipids and can be bridged by them," 
Nat. Struct. Mol. Biol., vol. 27, no. 11, 2020, doi: 10.1038/s41594-020-0494-5.

[73] H. Flores-Romero, U. Ros, and A. J. Garcia-Saez, "Pore formation in regulated cell death," EMBO J., 2020, doi: 10.15252/embj.2020105753.

[74] R. Salvador-Gallego et al., "Bax assembly into rings and arcs in apoptotic mitochondria is linked to membrane pores," $E M B O$ J., vol. 35, no. 4, pp. 389401, Feb. 2016, doi: 10.15252/embj.201593384.

[75] L. Große, C. A. Wurm, C. Brüser, D. Neumann, D. C. Jans, and S. Jakobs, "Bax assembles into large ring-like structures remodeling the mitochondrial outer membrane in apoptosis," EMBO J., vol. 35, no. 4, pp. 402-413, Feb. 2016, doi: 10.15252/embj.201592789.

[76] M. M. Cleland et al., "Bcl-2 family interaction with the mitochondrial morphogenesis machinery," Cell Death Differ., 2011, doi: 10.1038/cdd.2010.89.

[77] C. Brooks et al., "Bak regulates mitochondrial morphology and pathology during apoptosis by interacting with mitofusins," Proc. Natl. Acad. Sci. U. S. A., 2007, doi: 10.1073/pnas.0703976104.

[78] J. Prudent, R. Zunino, A. Sugiura, S. Mattie, G. C. Shore, and H. M. McBride, "MAPL SUMOylation of Drp1 Stabilizes an ER/Mitochondrial Platform Required for Cell Death," Mol. Cell, 2015, doi: 10.1016/j.molcel.2015.08.001.

[79] S. Montessuit et al., "Membrane Remodeling Induced by the Dynamin-Related Protein Drp1 Stimulates Bax Oligomerization," Cell, vol. 142, no. 6, pp. 889 901, Sep. 2010, doi: 10.1016/J.CELL.2010.08.017.

[80] A. Cassidy-Stone et al., "Chemical Inhibition of the Mitochondrial Division Dynamin Reveals Its Role in Bax/Bak-Dependent Mitochondrial Outer Membrane Permeabilization," Dev. Cell, 2008, doi: 10.1016/j.devcel.2007.11.019.

[81] A. Ruiz, E. Alberdi, and C. Matute, "Mitochondrial division inhibitor 1 (Mdivi-1) protects neurons against excitotoxicity through the modulation of mitochondrial function and intracellular Ca2+ signaling," Front. Mol. Neurosci., 2018, doi: 10.3389/fnmol.2018.00003.

[82] A. Korwitz et al., "Loss of OMA1 delays neurodegeneration by preventing stress-induced OPA1 processing in mitochondria," J. Cell Biol., 2016, doi: 10.1083/jcb.201507022.

[83] M. Giacomello and L. Pellegrini, "The coming of age of the mitochondria-ER contact: A matter of thickness," Cell Death and Differentiation. 2016, doi: 
10.1038/cdd.2016.52.

[84] X. Jiang, H. Jiang, Z. Shen, and X. Wang, "Activation of mitochondrial protease OMA1 by bax and bak promotes cytochrome c release during apoptosis," Proc. Natl. Acad. Sci. U. S. A., 2014, doi: 10.1073/pnas.1417253111.

[85] M. Karbowski, K. L. Norris, M. M. Cleland, S. Y. Jeong, and R. J. Youle, "Role of Bax and Bak in mitochondrial morphogenesis," Nature, 2006, doi: 10.1038/nature05111.

[86] A. Pyakurel, C. Savoia, D. Hess, and L. Scorrano, "Extracellular Regulated Kinase Phosphorylates Mitofusin 1 to Control Mitochondrial Morphology and Apoptosis," Mol. Cell, 2015, doi: 10.1016/j.molcel.2015.02.021.

[87] Y. Zaltsman et al., "MTCH2/MIMP is a major facilitator of tBID recruitment to mitochondria," Nat. Cell Biol., 2010, doi: 10.1038/ncb2057.

[88] S. Cogliati and L. Scorrano, "A BID on mitochondria with MTCH2," Cell Res., 2010, doi: 10.1038/cr.2010.100.

[89] S. B. Ma et al., "Bax targets mitochondria by distinct mechanisms before or during apoptotic cell death: a requirement for VDAC2 or Bak for efficient Bax apoptotic function," Cell Death Differ., vol. 21, no. 12, pp. 1925-1935, 2014, doi: 10.1038/cdd.2014.119.

[90] S. S. Roy, A. M. Ehrlich, W. J. Craigen, and G. Hajnóczky, "VDAC2 is required for truncated BID-induced mitochondrial apoptosis by recruiting BAK to the mitochondria," EMBO Rep., 2009, doi: 10.1038/embor.2009.219.

[91] S. Naghdi, P. Várnai, and G. Hajnóczky, "Motifs of VDAC2 required for mitochondrial Bak import and tBid-induced apoptosis," pp. 1-10, 2015, doi: 10.1073/pnas.1510574112.

[92] H. S. Chin et al., "VDAC2 enables BAX to mediate apoptosis and limit tumor development," Nat. Commun., 2018, doi: 10.1038/s41467-018-07309-4.

[93] H. V. Dudko, V. A. Urban, A. I. Davidovskii, and V. G. Veresov, "Structurebased modeling of turnover of Bcl-2 family proteins bound to voltagedependent anion channel 2 (VDAC2): Implications for the mechanisms of proapoptotic activation of Bak and Bax in vivo," Comput. Biol. Chem., 2020, doi: 10.1016/j.compbiolchem.2020.107203.

[94] S. Dadsena et al., "Ceramides bind VDAC2 to trigger mitochondrial apoptosis," Nat. Commun., vol. 10, no. 1, pp. 1-4, 2019, doi: 10.1038/s41467-019-096544. 
[95] S. Dadsena, D. G. Hassan, and J. C. M. Holthuis, "Unraveling the molecular principles by which ceramides commit cells to death," Cell Stress, vol. 3, no. 8, pp. 280-283, 2019, doi: 10.15698/cst2019.08.196.

[96] J. M. Gall et al., "Hexokinase regulates Bax-mediated mitochondrial membrane injury following ischemic stress," Kidney Int., vol. 79, no. 11, 2011, doi: 10.1038/ki.2010.532.

[97] J. G. Pastorino, N. Shulga, and J. B. Hoek, "Mitochondrial binding of hexokinase II inhibits Bax-induced cytochrome c release and apoptosis," J. Biol. Chem., 2002, doi: 10.1074/jbc.M109950200.

[98] S. P. Mathupala, Y. H. Ko, and P. L. Pedersen, "Hexokinase II: Cancer's double-edged sword acting as both facilitator and gatekeeper of malignancy when bound to mitochondria," Oncogene. 2006, doi: 10.1038/sj.onc.1209603.

[99] P. L. Pedersen, "Voltage dependent anion channels (VDACs): A brief introduction with a focus on the outer mitochondrial compartment's roles together with hexokinase-2 in the 'Warburg effect' in cancer," J. Bioenerg. Biomembr., 2008, doi: 10.1007/s10863-008-9165-7.

[100] M. N. Sohlang and S. Majaw, "Altered VDAC-HK association and apoptosis in mouse peripheral blood lymphocytes exposed to diabetic condition: an in vitro and in vivo study," Arch. Physiol. Biochem., 2021, doi: 10.1080/13813455.2020.1867187.

[101] J. Zhang, Y. Guo, W. Ge, X. Zhou, and M. Pan, "High glucose induces apoptosis of HUVECs in a mitochondria-dependent manner by suppressing hexokinase 2 expression," Exp. Ther. Med., 2019, doi: 10.3892/etm.2019.7609.

[102] K. Todkar, L. Chikhi, V. Desjardins, F. El-Mortada, G. Pépin, and M. Germain, "Selective packaging of mitochondrial proteins into extracellular vesicles prevents the release of mitochondrial DAMPs," Nat. Commun., vol. 12, no. 1, 2021, doi: 10.1038/s41467-021-21984-w.

[103] J. Wade Harper, A. Ordureau, and J. M. Heo, "Building and decoding ubiquitin hains for mitophagy," Nature Reviews Molecular Cell Biology, vol. 19, no. 2. 2018, doi: 10.1038/nrm.2017.129.

[104] S. R. Yoshii, C. Kishi, N. Ishihara, and N. Mizushima, "Parkin mediates proteasome-dependent protein degradation and rupture of the outer mitochondrial membrane," J. Biol. Chem., vol. 286, no. 22, 2011, doi: 10.1074/jbc.M110.209338. 
[105] S. A. Sarraf et al., "Landscape of the PARKIN-dependent ubiquitylome in response to mitochondrial depolarization," Nature, vol. 496, no. 7445, 2013, doi: 10.1038/nature12043.

[106] Z. Cakir et al., "Parkin promotes proteasomal degradation of misregulated BAX," J. Cell Sci., vol. 130, no. 17, pp. 2903-2913, Sep. 2017, doi: 10.1242/jcs.200162.

[107] R. A. Charan, B. N. Johnson, S. Zaganelli, J. D. Nardozzi, and M. J. LaVoie, "Inhibition of apoptotic Bax translocation to the mitochondria is a central function of parkin," Cell Death Dis., vol. 5, no. 7, 2014, doi: 10.1038/cddis.2014.278.

[108] G. Dewson et al., "To Trigger Apoptosis, Bak Exposes Its BH3 Domain and Homodimerizes via BH3:Groove Interactions," Mol. Cell, 2008, doi: 10.1016/j.molcel.2008.04.005.

[109] J. P. Bernardini et al., "Parkin inhibits BAK and BAX apoptotic function by distinct mechanisms during mitophagy," EMBO J., vol. 38, no. 2, 2019, doi: 10.15252/embj.201899916.

[110] Y. Fernández-Marrero, S. Bleicken, K. K. Das, D. Bachmann, T. Kaufmann, and A. J. Garcia-Saez, "The membrane activity of BOK involves formation of large, stable toroidal pores and is promoted by cBID," FEBS Journal. 2017, doi: 10.1111/febs. 14008 .

[111] J. H. Zheng et al., "Intrinsic Instability of BOK Enables Membrane Permeabilization in Apoptosis," Cell Rep., vol. 23, no. 7, 2018, doi: 10.1016/j.celrep.2018.04.060.

[112] F. Llambi et al., "BOK Is a Non-canonical BCL-2 Family Effector of Apoptosis Regulated by ER-Associated Degradation," Cell, 2016, doi: 10.1016/j.cell.2016.02.026.

[113] S. Einsele-Scholz et al., "Bok is a genuine multi-BH-domain protein that triggers apoptosis in the absence of Bax and Bak," J. Cell Sci., vol. 129, no. 11, pp. 2213-2223, Jun. 2016, doi: 10.1242/jcs.181727.

[114] J. J. Schulman, F. A. Wright, T. Kaufmann, and R. J. H. Wojcikiewicz, "The Bcl2 protein family member bok binds to the coupling domain of inositol 1,4,5trisphosphate receptors and protects them from proteolytic cleavage," J. Biol. Chem., vol. 288, no. 35, pp. 25340-25349, Aug. 2013, doi: 10.1074/jbc.M113.496570. 
[115] M. A. Carpio, R. E. Means, A. L. Brill, A. Sainz, B. E. Ehrlich, and S. G. Katz, "BOK controls apoptosis by $\mathrm{Ca} 2+$ transfer through ER-mitochondrial contact sites," Cell Rep., vol. 34, no. 10, 2021, doi: 10.1016/j.celrep.2021.108827.

[116] N. Echeverry, D. Bachmann, F. Ke, A. Strasser, H. U. Simon, and T. Kaufmann, "Intracellular localization of the BCL-2 family member BOK and functional implications," Cell Death Differ., vol. 20, pp. 785-799, 2013, doi: 10.1038/cdd.2013.10.

[117] G. Basañez et al., "Bax, but not Bcl-XL, decreases the lifetime of planar phospholipid bilayer membranes at subnanomolar concentrations," Proc. Natl. Acad. Sci. U. S. A., vol. 96, no. 10, 1999, doi: 10.1073/pnas.96.10.5492.

[118] M. Lutter, M. Fang, X. Luo, M. Nishijima, X. S. Xie, and X. Wang, "Cardiolipin provides specificity for targeting of tBid to mitochondria," Nat. Cell Biol., 2000, doi: 10.1038/35036395.

[119] A. Shamas-Din, S. Bindner, X. Chi, B. Leber, D. W. Andrews, and C. Fradin, "Distinct lipid effects on tBid and Bim activation of membrane permeabilization by pro-apoptotic Bax," Biochem. J., 2015, doi: 10.1042/bj20141291.

[120] S. Lucken-Ardjomande, S. Montessuit, and J. C. Martinou, "Contributions to Bax insertion and oligomerization of lipids of the mitochondrial outer membrane," Cell Death Differ., vol. 15, no. 5, pp. 929-937, May 2008, doi: 10.1038/cdd.2008.9.

[121] O. Landeta, A. Landajuela, A. Garcia-Saez, and G. Basañez, "Minimalist model systems reveal similarities and differences between membrane interaction modes of MCL1 and BAK," J. Biol. Chem., vol. 290, no. 27, pp. 17004-17019, Jul. 2015, doi: 10.1074/jbc.M114.602193.

[122] H. Flores-Romero et al., "BFL1 modulates apoptosis at the membrane level through a bifunctional and multimodal mechanism showing key differences with BCLXL," Cell Death Differ., vol. 26, no. 10, pp. 1880-1894, Oct. 2019, doi: 10.1038/s41418-018-0258-5.

[123] M. E. Maes, J. A. Grosser, R. L. Fehrman, C. L. Schlamp, and R. W. Nickells, "Completion of BAX recruitment correlates with mitochondrial fission during apoptosis," Sci. Rep., vol. 9, no. 1, 2019, doi: 10.1038/s41598-019-53049-w.

[124] P. Wang et al., "Dynamin-related protein Drp1 is required for Bax translocation to mitochondria in response to irradiation-induced apoptosis," Oncotarget, vol. 6, no. 26, 2015, doi: 10.18632/oncotarget.4200. 
[125] J. E. Chipuk et al., "Sphingolipid Metabolism Cooperates with BAK and BAX to Promote the Mitochondrial Pathway of Apoptosis," Cell, vol. 148, no. 5, pp. 988-1000, 2012, doi: 10.1016/j.cell.2012.01.038.

[126] H. Birbes, C. Luberto, Y.-T. Hsu, S. EL Bawab, Y. A. Hannun, and L. M. Obeid, "A mitochondrial pool of sphingomyelin is involved in TNFa-induced Bax translocation to mitochondria," Biochem. J., 2005, doi: 10.1042/bj20041627.

[127] M. Charman, B. E. Kennedy, N. Osborne, and B. Karten, "MLN64 mediates egress of cholesterol from endosomes to mitochondria in the absence of functional Niemann-Pick Type C1 protein," J. Lipid Res., vol. 51, no. 5, pp. 1023-1034, May 2010, doi: 10.1194/jr.M002345.

[128] K. Bartel et al., "Connecting lysosomes and mitochondria - A novel role for lipid metabolism in cancer cell death," Cell Commun. Signal., vol. 17, no. 1, pp. 116, Jul. 2019, doi: 10.1186/s12964-019-0399-2.

[129] K. Bartel et al., "V-ATPase inhibition increases cancer cell stiffness and blocks membrane related Ras signaling - A new option for HCC therapy," Oncotarget, vol. 8, no. 6, pp. 9476-9487, Dec. 2017, doi: 10.18632/oncotarget.14339.

[130] C. D. DiNardo et al., "Venetoclax combined with decitabine or azacitidine in treatment-naive, elderly patients with acute myeloid leukemia," Blood, vol. 133, no. 1, pp. 7-17, Jan. 2019, doi: 10.1182/blood-2018-08-868752. 


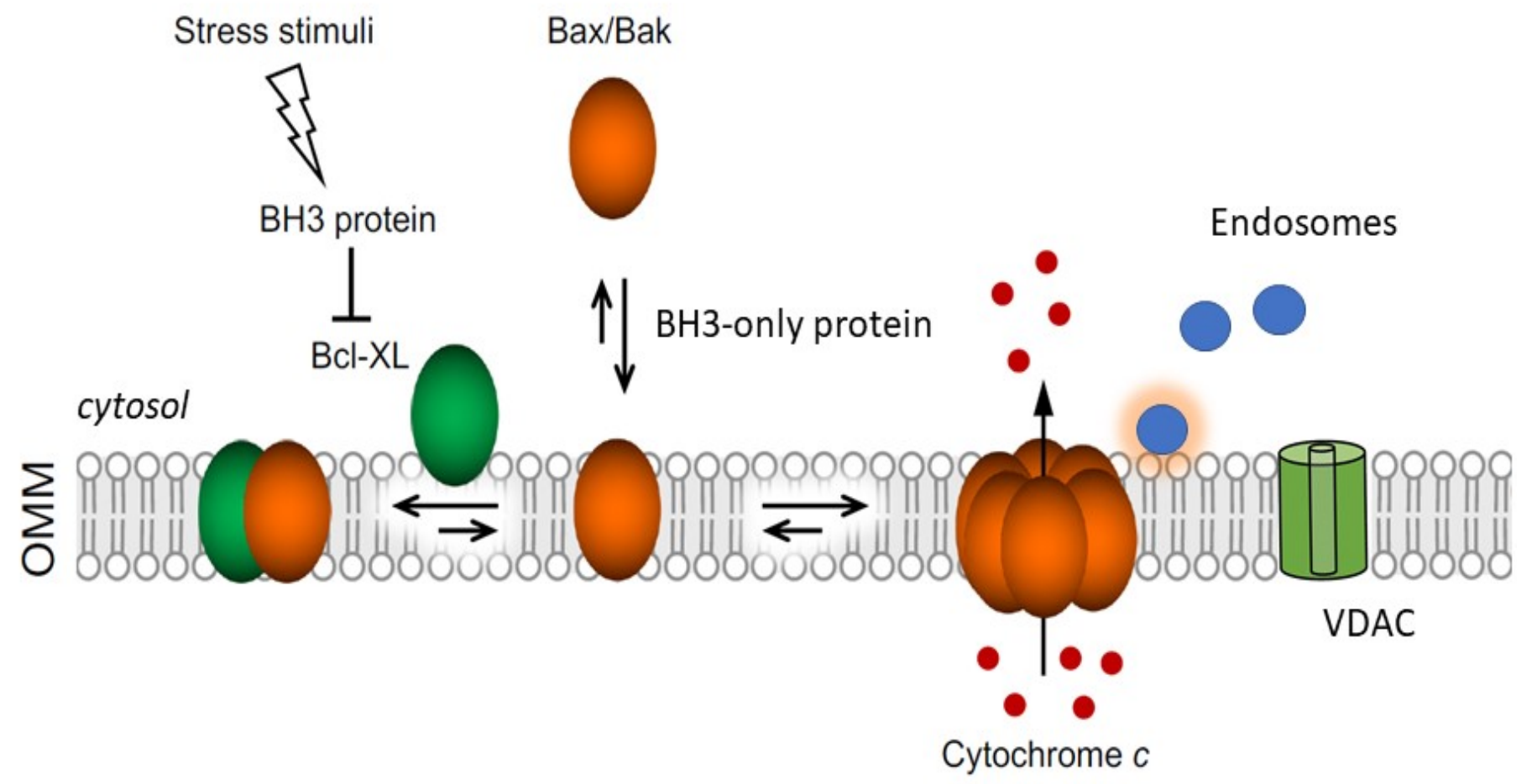

Graphical Abstract 


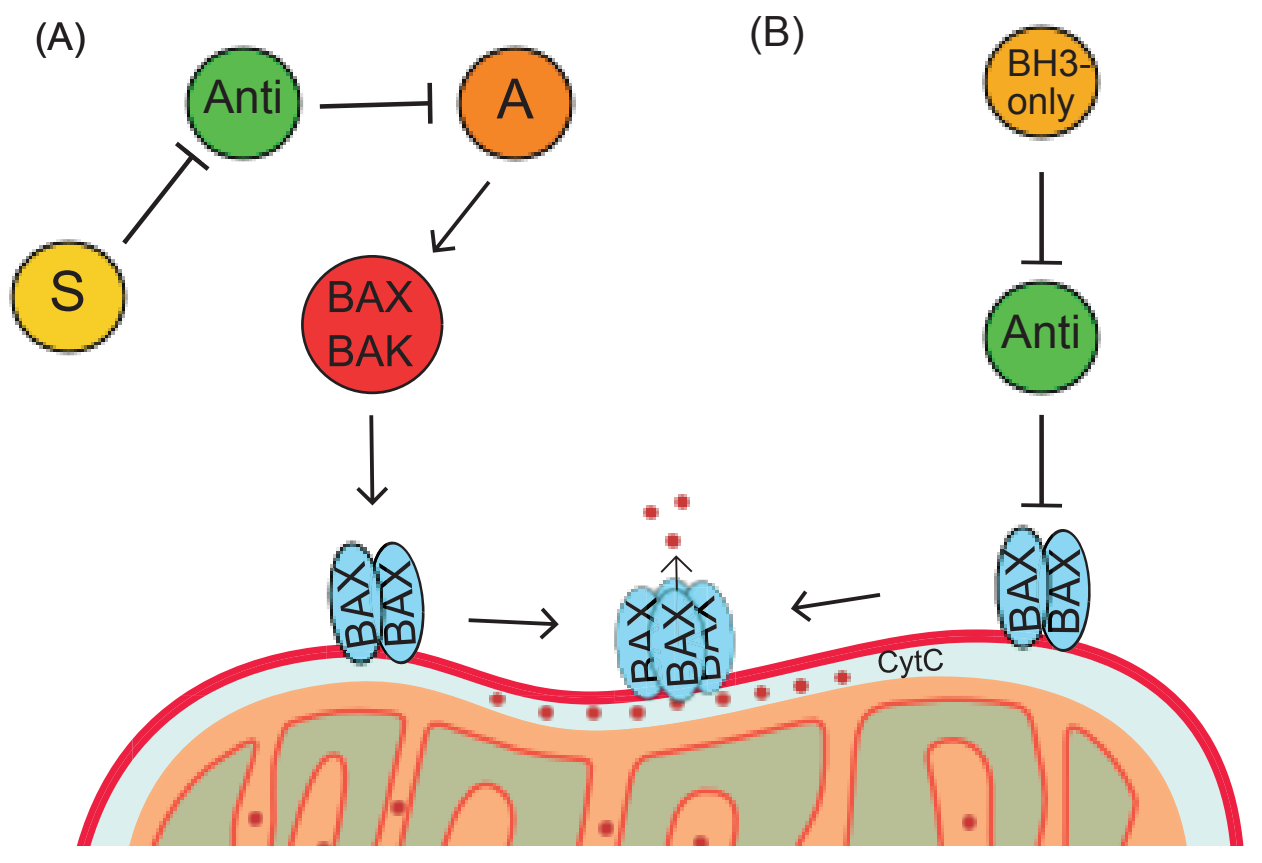

(C) -1 BAX

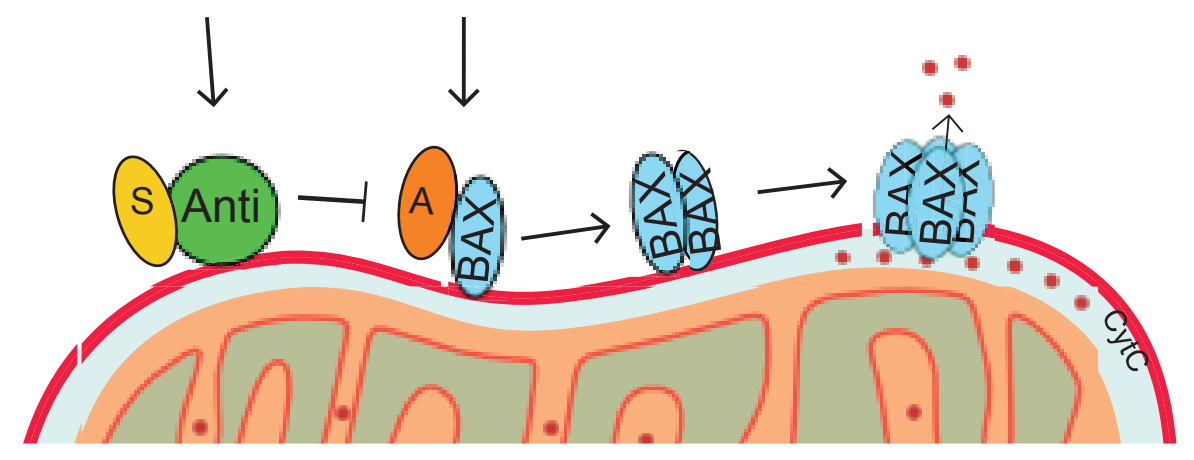

Figure 1 


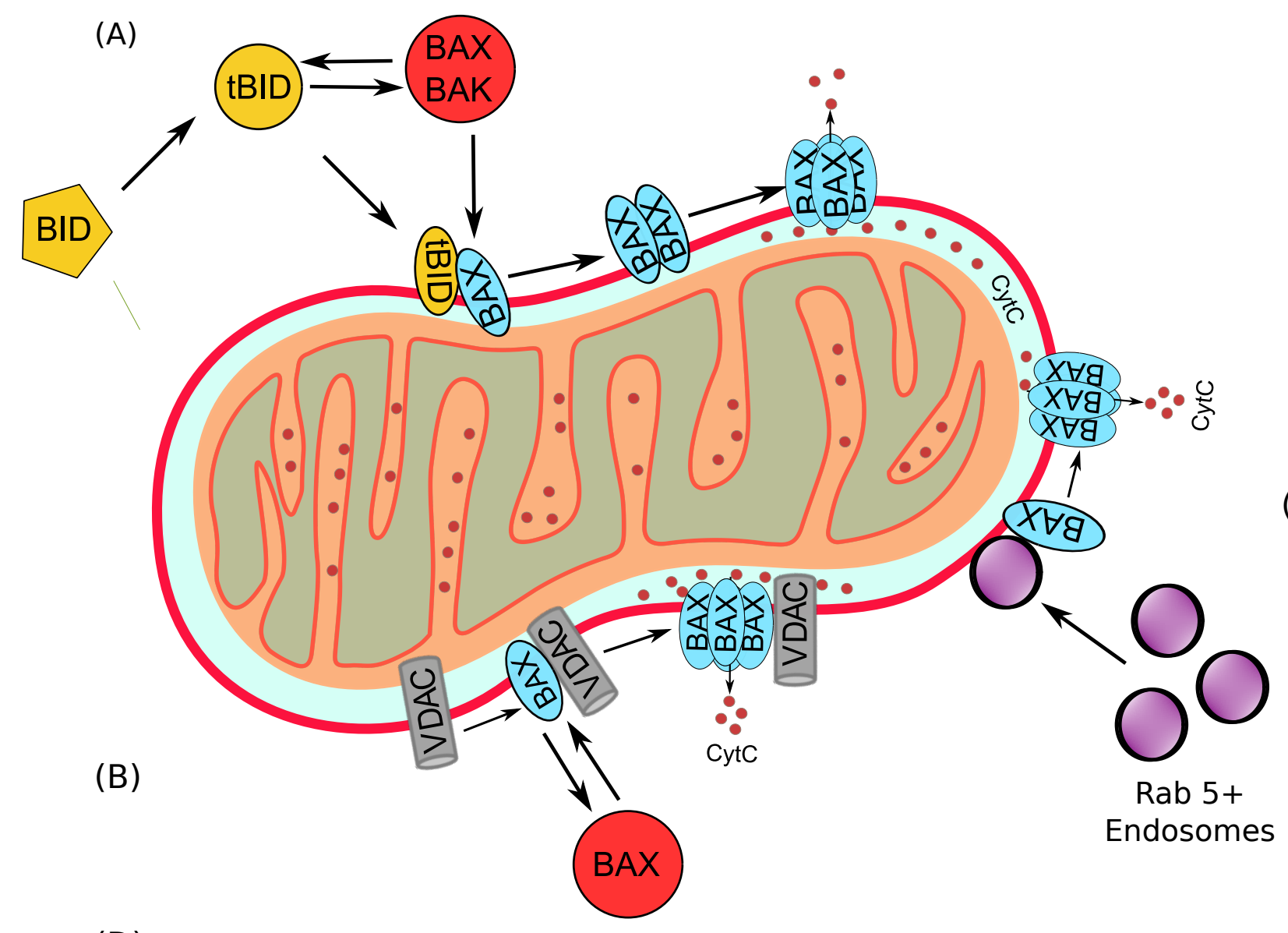

(C)

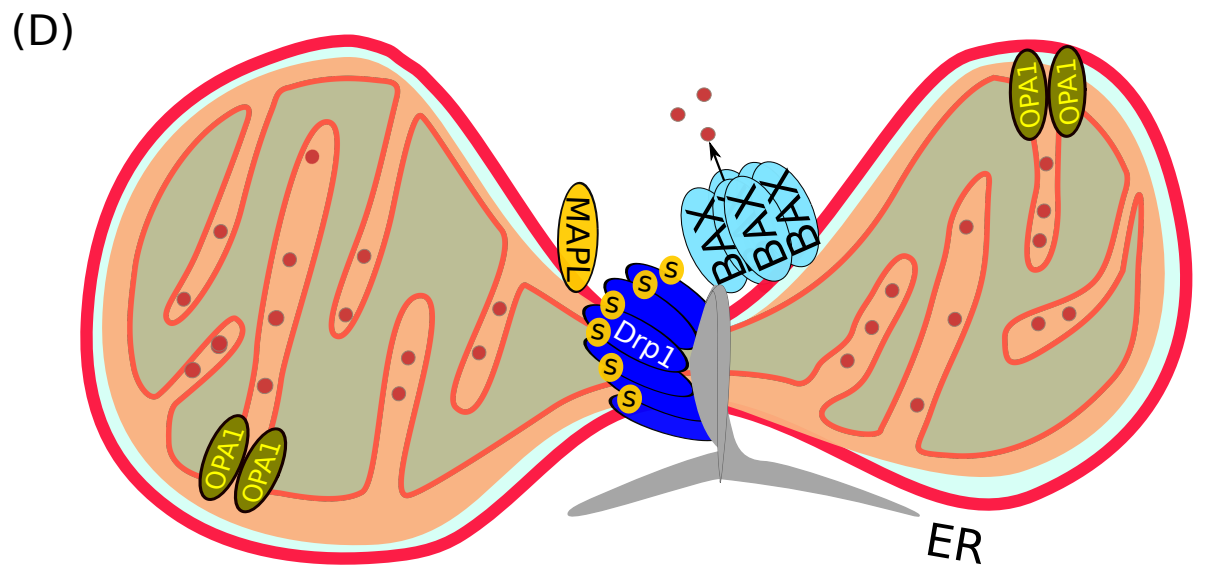

Figure 2 